\title{
Psychiatric Presentations To Primary Care
}

\author{
NJ Thomson
}

\section{Introduction}

Psychiatric morbidity is a common theme in primary care. Twenty five percent of all attenders, $52 \%$ of high utilisers and $46 \%$ of illness initiation visits in primary care have a diagnosable psychiatric illness (1). Research demonstrates that detection rates are poor with up to $50 \%$ of cases presenting being missed (2). Some of these cases will be minor self-limiting conditions which improve spontaneously. Others will present with physical symptoms, finding it difficult to use emotional vocabulary. It is also true that time constraints in primary care are not conducive to full and detailed exploration of symptom profiles which may be complicated and difficult to elicit.

The majority of psychiatric morbidity seen in the General Practice surgery does not constitute an emergency but all has the potential to generate a significant amount of anxiety in the clinician. This anxiety may be fuelled by concerns about risk of harm, by media speculation or simply by lack of familiarity.

The following text aims to highlight common presentations, to suggest underlying formulations and to advise on management. Symptoms and signs however, are inevitably coloured by personality and circumstance. Presentations therefore have the potential to be as varied as the patients themselves.

\section{Presenting Symptons}

\section{Physical symptoms}

The relationship between physical and psychiatric symptomatology is a complicated one. Both sets of symptoms may be present coincidentally, although it would be unusual for neither to have an affect on the other. Both may be present, colouring the presentation of the other as is seen with pain and low mood. Here the pain contributes to the low mood which in turn reduces the tolerance to pain perception. Physical disease may result in psychiatric symptoms as is seen in encephalopathies, endrocrinopathies and Central Nervous System (CNS) tumours. Finally, psychiatric disorder may result in the presentation of only physical symptoms.

Until recently, psychiatric disorders presenting with only physical symptoms were generally referred to as hysteria.
Symptoms occur in the absence of physical pathology, and are produced unconsciously rather than deliberately. They reflect the patient's knowledge of illness and so may not conform to the expected presentation. An example would be a pattern of anaesthesia which does not conform to anatomical innervation. They usually confer some advantage on the patient although advantage as a result of illness may also be seen in physical and other psychiatric disorders. Disorders of movement such as paralysis and tremor are seen, as well as disorders of sensation such as anaesthesia and pain.

A psychogenic aetiology is suggested by a younger age of presentation, as these disorders seldom appear for the first time after the age of 40 . They are often provoked by stressful events and symptoms can be seen to be advantageous in some way. The last two indicators are not exclusive to these disorders however, and so do not constitute very specific criteria.

\section{Psychosis}

Psychotic symptoms are relatively rare in general practice although such symptoms are indicative of serious mental illness and much associated suffering. A range of severity is seen, from a florid chaotic and confused picture, through to a much more subtle and difficult to elicit presentation. The hallmarks of psychotic disorder include delusional ideas and hallucinations. Delusions are beliefs which are fixed and held with $100 \%$ certainty, and which are held on inadequate grounds. They are unaffected by rational argument or evidence to the contrary and they are not considered conventional given the patient's educational and cultural background. Of note, this is not to say that these beliefs are always false. Hallucinations may occur in any sensory modality and may be defined as a sensory percept in the absence of any stimulus. In other words, the patient experiences sights, sounds and sensation in a qualitatively normal way, without the relevant visual auditory or tactile stimulus.

The symptom which often makes the psychotic patient difficult to interview is that of formal thought disorder. At the mild end of the spectrum thoughts are difficult to follow with obscure or tenuous links between topics. At the other end, speech is 
unintelligible and reflects thoughts which have no associations with each other. Words may be used inappropriately or new meaningless words made up (neologisms).

Whilst it may be relatively straightforward to formulate a working diagnosis for a floridly psychotic patient, quite often the General Practitioner (GP) is presented with a far subtler picture of change in performance and deterioriation in interpersonal relationships. Detailed and sensitive interviewing is required to build the trust necessary to disclose any frightening, odd or stigmatising symptoms.

\section{Abnormal Mood}

Disturbance in mood is probably the most frequently encountered psychiatric symptom in general practice and may present as elevation or lowering of mood. Elevation of mood is rarely the primary complaint of a patient but may be accompanied by irritability, distractibility or racing thoughts. Quite often these patients are ordered to attend the medical centre due to the social chaos that inevitably follows their clinical condition. Sleep is often poor but associated with the perception that less is required. Appetite is increased without weight gain and feelings of having unlimited supplies of energy are common. Concentration is often poor with thoughts flitting from one topic to the next with little continuity. This may be reflected in speech, which is pressured and sometimes difficult to follow. Poor decisions are made, money is spent unwisely and relationships are entered into and broken off repeatedly. The act of simply interviewing such a patient can render the interviewer confused and fatigued!

Lowering of mood is more common. Prevalence of depressive disorder ranges from 2.3 to $8.7 \%$ (3) although depressive symptoms may be seen in as much as $20 \%$ of the general population (4). Low mood may be described as unhappiness, depression or distress. It may be present constantly, variably or only in response to certain stressors. Biological and or cognitive symptoms add to the clinical picture. Biological, or vegetative, symptoms consist of poor sleep with initial insomnia, a broken pattern or early morning waking; poor appetite, loss of concentration, anergia and anhedonia. Cognitive symptoms include feelings of hopelessness, helplessness and worthlessness. Suicidal ideation may be the presenting symptom and if not, should always be enquired after. There is no evidence that asking patients about suicidal thoughts increases the likelihood of a suicide attempt (5), although enquiries do need to be made with a sense of empathy and understanding. The intensity and pervasiveness of such thoughts aid in the assessment of risk as does evidence of planning, preparation and previous acts.

\section{Neurosis}

In the past, anxiety has been classified with the mood disorders of depression and elation. Today it is most commonly included in the neurotic disorders, the definition of which specifies that they are not accompanied by organic brain disease, that they are not psychoses regardless of severity, and that they differ from personality disorder in having a discrete as opposed to a continuous onset. This category comprises of anxiety and obsessional disorders, minor affective disorders and adjustment reactions.

Anxiety is a physiological and cognitive condition which may be abnormal if pervasive or if in response to stimuli which would not usually be considered to be anxiety provoking. Somatic symptoms consist of increased heart rate, light headedness, dizziness, increased respiratory rate, pins and needles especially in extremities, nausea, frequency of urine and any other physical symptoms consistent with the 'fight or flight' response. Cognitive symptoms include a sense of fearfulness or panic with possible concerns about fainting, being sick or dying. This unpleasant affect often leads to avoidance of the triggering stimuli, therefore strengthening the stimulus-response relationship. When an individual has witnessed or been part of a traumatic event, poor memory processing of that event may result in Post Traumatic Stress Disorder. In this condition cognitive and somatic symptoms of anxiety are seen in response to reminders of the event and avoidance of these reminders is often extreme. As well as avoidance, arousal, distress in response to reminders and reliving phenomena such as flash backs and nightmares, there is often a sense of emotional detachment. Common comorbidity includes depressive disorders and substance misuse.

Obsessional symptoms are words, ideas or beliefs recognised by the patient as his own, that intrude forcibly into his mind. They are usually unpleasant and so are often resisted. They may develop into ruminations, doubts, impulses or rituals and become linked with a compulsion i.e. a behavioural ritual whose function, at least initially, is to combat the obsessional thinking. An example would be ruminations about dirt leading to compulsive hand washing.

Mild and transient symptoms of low mood, anxiety, irritability and poor concentration are sometimes seen in patients previously free of mental disorder. They are related closely in time and content to a perceived stressor and subside once the stress resolves.

Substance Misuse

Substance misuse and its related 
problems are a common presentation to the GP and one that generates strong feelings in patient, Doctor and Commanding Officer alike. Gone are the days when alcohol was the only substance one needed to be concerned about; with up to $25 \%$ of all adolescents having at least experimented with illicit drugs (6), screening for misuse of all substances is indicated.

Presentation is once again variable from a relatively straightforward picture of intoxication to the insidious onset of a range of biopsychosocial problems. Multiple entries into medical notes indicating assaults, accidents and regular intoxication should raise the index of suspicion. Difficulties with impulse control and complaints of chronic abnormal mood states may also add to the aetiological picture. Social chaos with relationship, forensic and financial difficulties may also point to an underlying substance misuse problem. Information is often unforthcoming and therefore screening is necessary. A non-judgemental, and where possible non-punitive approach often improves detection in this field.

\section{Self-harm}

Since the 1950s, a distinction has been made between those who kill themselves and those who survive an apparent suicide attempt. "Attempted suicide", "parasuicide", and "deliberate self-harm" (DSH) have all been used to describe the latter group. There is a danger, however, in assuming that all who fail at suicide do not display any real intent, or that all who complete suicide desire death as the outcome. These two groups overlap with each other, and with a third group of individuals who remain ambivalent, uncertain if they want to live or die. It must be remembered that among those who deliberately self-harm, the completed suicide rate in the subsequent twelve months is 100 times greater then in the general population (7).

Threats or acts of self-harm are among the more common psychiatric presentation to primary care. In the United Kingdom, about $90 \%$ of the cases of DSH referred to general hospitals involve drug overdose (5); the most common drugs used being anxiolytics and non-opiate analgesics. The use of paracetamol is increasing due in part to its ready availability. Self-laceration is another common method, usually consisting of superficial wounds to wrists and forearms.

Important history to note includes the subjective belief about the severity of the act, any reported wish to die and subsequent feelings once the act has failed to cause death. Prior thoughts, plans and intent should be explored as well as any continuing wish to self-harm.
Circumstances surrounding or triggering the attempt are important, as well as any subsequent changes in the perception of them. Detection of any mental illness is a necessary part of risk assessment and clinical management. In general whilst highlighting the dangers of assumption as above, a high risk of completed suicide is associated with planning in advance, precautions to avoid discovery, no attempts to obtain help afterwards, dangerous methods, the presence of mental disorder and previous suicide attempts.

\section{Formulations}

Organic disorder as a cause of mental illness must always be considered. Acute confusional states may be seen in alcohol or drug intoxication or withdrawal. More chronic substance misuse problems may result in a psychotic or dementing picture. Primary injury or insult affecting the brain directly may be more easily detected, but more systemic diseases such as thyroid disease should also be considered. Side effects of prescribed and non-prescribed medication are another important cause of psychiatric symptoms.

Substance misuse diagnoses fall into the categories of intoxication, harmful use and dependence syndrome. A diagnosis of harmful use implies that there is a pattern of psychoactive substance use which is causing damage to physical or psychological health. Dependence syndrome includes a cluster of physiological behavioural and cognitive symptoms in which use of the substance takes on a much higher priority for a given individual than other behaviours that once had greater value. There is a sense of compulsion to take the substance, tolerance develops to the effects of it, and physiological withdrawal symptoms are seen on cessation of the substance. Dependence syndrome may be present for a specific substance, for a class of drugs or for a wider range of difference drugs.

Psychotic symptoms indicate the presence of one of several disorders. Schizophrenia is often the first diagnosis considered although it should be remembered that such a label has a powerfully stigmatising effect. As such the criteria for diagnosing schizophrenia are detailed and tightly defined and can be found in the International Classification of Diseases, Classification of Mental and Behavioural Disorders. A severe depressive or manic illness may present with psychosis, the flavour of the symptoms reflecting the underlying mood disorder. Psychosis may result from an acutely stressful experience although the main body of research indicates that substantial proportion of these cases go on to develop into a schizophreniform or affective psychosis (8). Some symptoms may be outside the bounds 
of normal experience yet not reach the definition for symptoms of psychosis. An example would be hearing a voice which appears to be originating in one's own head. This may indicate a developing or resolving full psychotic picture or one of the many symptoms experienced in personality disorder.

Depressive episodes are categorised into mild, moderate and severe. Central elements such as low mood, anergia and anhedonia are accompanied by biological and cognitive symptoms as previously mentioned. Manic episodes may also be mild (hypomania), moderate or severe, and it is often the behavioural aspect of this disorder which is most striking. The rate of speech, thoughts and behaviour is increased and the overall picture is one of rapid chaotic thinking and behaviour, often associated with evidence of grandiosity and poor judgement. In its most severe form the patient is at risk from becoming completely mentally and physically exhausted.

Neurotic disorders are numerous and varied. A diagnosis of phobic disorder implies a rapid onset of severe and disabling anxiety in response to an identified and specific trigger leading to avoidance of that trigger. Panic disorder may consist of a similar clinical picture but no identifiable trigger is seen. Episodes of panic have a rapid onset, are associated with somatic symptoms and cognitive symptoms of anxiety and rarely last for more than a few minutes. Generalised anxiety disorder is another diagnosis to consider when the anxiety is the predominant symptom. The picture is more pervasive and "free floating" however, leading to milder but constant debilitation. Obsessive compulsive disorder describes a syndrome of obsessive thoughts and compulsive behaviour. When severe the cognitive and behavioural rituals may take so long to complete that the patient is seen to be suffering from obsessional slowness. The presentation of only physical symptoms in the absence of any organic pathology is indicative of somatization disorders. Mild and transient disturbance in mood in relation to perceived stressors are termed Adjustment Disorders and usually have a good prognosis with removal of the perceived stressor.

Chronic patterns of maladaptive behaviour and disturbed interpersonal relationships associated with subjective distress and impaired functioning are termed Personality Disorder. To make this diagnosis these patterns should have been present from late teenage years and often will require confirmation by third party historians. Care is required when considering such a diagnosis as, once again, it is a debilitating and stigmatising label. Different subcategories of Personality Disorder are associated with different patterns of behaviour, thinking and relationship difficulties, and whilst there is some evidence to suggest character traits "mellow" with age, short to medium prognosis is often poor. Presenting symptoms are as varied as the problems these patients encounter although $\mathrm{DSH}$, substance misuse and interpersonal conflict are common themes.

\section{Management}

Assessment needs to be as comprehensive and as detailed as possible. Past and present history helps to build up a holistic picture of the patient and third party history is often invaluable. Physical examination and where possible, blood screening and drug urinalysis help to clarify the picture and in the formulation of differential diagnoses.

Risk assessment is often a necessary element of initial assessment. There may be a risk of harm to self, to others or of deterioration in physical or psychological health. There is no infallible way to accurately predict this risk but a previous history of harm significantly raises the index of suspicion.

Treatment of mental illness is most usefully considered within a biopsychosocial model. Physical therapies include treatment of organic illness, psychoactive medication, ECT and psychosurgery. The range of antipsychotics, antidepressants, anxiolytics and sedatives is extensive, and it is usually appropriate to prescribe familiar drugs with evidence based research demonstrating their effectiveness. Side effects and potential for dependence and overdose must always be considered.

It is important to have to hand medication that may be needed in an emergency, such as aggressive behavioural disturbance. An antipsychotic such as droperidol or haloperidol may be indicated to settle a psychotic presentation, whilst a short acting benzodiazepine is used to sedate and treat the behavioural disturbance. Oral medication is preferable, however intramuscular dosing may be necessary if risk is high and insight is lacking. If this is the case, medication is administered under common law and following this, assessment for detention under the Mental Health Act should be made as soon as possible.

Psychological management may consist of supportive psychotherapy carried out by empathic clinicians who do not necessarily have any formal training, through to cognitive behavioural therpay better undertaken by psychologists. Social management is also important to consider as social situation and psychiatric pathology are often intimately related. Housing, finances, activities and relationships may all be amenable to manipulation which in isolation, can lead to an improvement in 
clinical condition.

Advice from specialists and onwards referral are appropriate considerations when the presenting case is complex, appears to be treatment resistant or is associated with a significant risk of harm. The threshold for admission to the Duchess of Kent's Psychiatric Hospital (DKPH) is lower than that to National Health Service mental health units, due mainly to the requirement for specialised occupational management. Assessment is offered for any referred serviceman who appears to have psychological difficulties and for whom admission may be considered. If admitted, further assessment and treatment are managed by a multidisciplinary team, including psychologist, social worker and occupational therapist input. Following assessment, clinicians aim to provide the GP with a diagnostic and prognostic statement as well as occupational recommendations. If the patient has been assessed as having a temperament unsuited to military service, a recommendation for administrative discharge may be forthcoming. A mental disorder with good medium and long term prognosis may result in a recommendation to temporarily downgrade, and one with a poor prognosis may result in a recommendation to medically discharge.

The nature of mental disorder is such that assessment may often be protracted, taking a matter of weeks rather than days. This process is often aided by third party history such as GP history, F Med 8 and conduct sheets. On discharge from hospital, and where indicated, clinical condition, medical grading and medication is monitored at outpatient appointments. The aim, as in all areas of military medicine, is to protect the individual and the service during this period of rehabilitation with a view to returning him to full and active duty.

\section{Conclusion}

Mental health problems are a common theme in primary care, and range in severity from the mild and episodic through to the chronically disabling. Signs and symptoms are often frightening and bewildering to both sufferer and onlooker. The majority of mental disorder is amenable to treatment, although recovery may be protracted, and often hampered by the attitudes of collegues, friends and family. Successful management relies heavily on detection of difficulties, and whilst patients may often appear angry, aggressive or manipulative, this facade often hides real and significant emotional problems. Forming a therapeutic relationship to allow detailed exploration of these difficulties and subsequent application of focused treatment modalities is a challenging and often rewarding achievement. Consideration is given to the patient's physical and psychological wellbeing, as well as to his interaction with the world around him. The treatment of emotional problems almost always has an impact on all of these facets of the patient's life.

\section{References}

1. Bowers PJ. Selections from current literature: psychiatric disorders in primary care. Family Practice 1993; 10(2): 231-237

2. Blacker CVR. Clare AW. Depressive disorder in primary care. British fournal of Psychiatry 1987; 150: 737

3. Robins LN, Helzer JE, Weissman MM. Lifetime prevalence of specific psychiatric disorders in three sites. Archives of General Psychiatry 1984; 41: 949958

4. Boyd JH, Weissman MM. Epidemiology. In: Handbook of Affective Disorders. Churchill Livingstone, Edinburgh, 1982

5. Gelder M, Gath D, Mayou R, Cowen P. Mental State Examination In: Oxford textbook of Psychiatry, 3rd edn. Oxford Medical Publications, 1996; 34

6. Leitner M, Shapland J, Wiles P. What kind of people use drugs? In: Drug usage and drugs prevention, the views and habits of the general public. Home Office Drugs Prevention Initiative, 1993; 24

7. Kreitman N, Casey P. The repetition of parasuicide; an epidemiological and clinical study British Fournal of Psychiatry 1988; 153: 792-800

8. Bergen ALM, Dahl AA, Guldber C, Hansen H. Langfelt's schizophreniform psychosis fifty years later. British Fournal of Psychiatry 157: 351-354 